Bol. Acad. peru. leng. 48. 2009 (75-89)

\title{
LOS SENTIDOS CORPORALES EN MOCHICA
}

\author{
LES SENS CORPORELS DANS MOCHICA
}

THE CORPORAL SENSES IN MOCHICA

\author{
José Antonio Salas García
}

Resumen:

El objetivo del presente artículo es traducir del mochica al castellano el texto correspondiente a los sentidos corporales que aparece en el Arte de la lengua yunga de Fernando de la Carrera (1644). Como acontece con otros textos mochicas que hemos tenido ocasión de interpretar (Salas 2008), los resultados de la traducción nos muestran textos que no son exactamente iguales en su contenido a las versiones que existen de estas oraciones en castellano.

Résumé:

Le présent article a pour objet la traduction du moche vers l'espagnol du texte portant sur les sens corporels de l'Arte de la lengua yunga de Ferdinand de la Carrera (1644). Tout comme dans le cas de textes moches que nous avons eu l'occasion d'interpréter (Salas 2008), la traduction qui en résulte est la preuve que le contenu de ces textes ne correspond pas entièrement aux versions espagnoles de ces prières. 


\section{Abstract:}

The aim of the present article is to translate from the mochica to Castilian the text corresponding to the corporal senses that appears in the Arte de la lengua yunga of Fernando de la Carrera (1644). As it happens with other mochica texts that we have tried to interpret (Salas 2008), the results of the translation show us texts that are not exactly equal in their content to the versions that exist of these (prayers) in Spanish.

Palabras clave:

Lengua mochica; traducción; textos cristianos.

Mots clés :

Langue moche; traduction; textes chrétiens.

Key words:

Mochica language; translation; Christian texts.

Fecha de recepción:

$20 / 07 / 2009$

Fecha de aceptación:

$15 / 08 / 2009$

\section{Introducción}

Los textos existentes de la lengua mochica corresponden a dos fuentes del siglo XVII: Oré 1607 y Carrera 1644. Ambos comparten la característica de haber aparecido en la imprenta sin una traducción al castellano. Al parecer, ninguno de los autores asumió que la lengua iba a desaparecer sin dejar un solo hablante. La traducción comporta problemas que se derivan del hecho de que los textos en mochica no siguen de manera estricta, las oraciones cristianas, tal como lo había estipulado el Tercer Concilio Limense. En efecto, la mayoría de textos corresponde a la Doctrina Christiana de 1584. Sin embargo, De la Carrera se tomó una licencia e intercaló en los textos de la Doctrina, uno que no correspondía a esta obra. Se trata de un pequeño fragmento que versa sobre los sentidos corporales. Nuestro propósito es dar una traducción razonada de este texto, tal como lo hicimos en Salas 2008b. 


\section{Corpus}

El corpus que toca analizar está compuesto por la descripción de los cinco sentidos y un pequeño diálogo acerca de ellos. Hemos copiado estos textos tal como aparecen en la gramática de Fernando de la Carrera (1644: 246):

Exll mætzhofær quicær chiçær lecuo çiofe: ñoc çæc.

Pac xang ñico çiofe: næmaçæc çoc xang nico çiofe: Oizçæc.

Noc xang nico çiofe: ssapæeco cu tzho chiçæc.

Exll mætzh xang nico çiofe: cof çæc.

Sobre los sentidos corporales

P. Ech æng, ame mo exll mætzho ær quic ær chiçær?

R. Mo ærquic ær chi çær fe: lock ero aqui çæc: aieçæn fe, med quic ero næma çæc: aieçæn fe, fon ero oiz çæc: aieçæn fe, ssapæco cutzho chi çæ: aieçæn fe mæcuaro cof çæc.

P. Ech pænoix piycæ mædo Dios i mo exll mætzho ærquicær chiçær?

R. Izçæcna çiong er Dios mæcha læcnæm, çio çæn seruir læcnæm.

\section{Metodología}

Para analizar el corpus, hemos optado por segmentar las dicciones con arreglo a los siguientes criterios: 1) La división entre palabras se hará teniendo en cuenta los lexemas existentes en la frase. Así, si en el texto original se lee: $\langle c ̧ i o f e\rangle$ que presenta unidos al pronombre de tercera persona çio con el verbo copulativo $f e$, entonces, nosotros transcribiremos: 〈çio fe〉, por tratarse de dos lexemas diferentes. Del mismo modo, siguiendo el criterio del lexema como base, el numeral $\langle$ Exll mætzh $\rangle$ que representa al número 'cinco' será transcrito como $\langle$ Exllmætzh $\rangle$, por tratarse de un solo lexema y no de dos. 2) Los lexemas que contengan morfología susceptible de análisis estarán segmentados por un guión medio. De esta manera, si en medquic, que es la forma no poseída del concepto 'oreja' encontramos una raíz med y un morfema de no posesión -quic, entonces, el lexema 
será transcrito como med-quic. 3) Los clíticos y las frases posposicionales se segmentarán mediante un guión bajo. Ejemplo: Al nombre med-quic 'oreja' unido a la posposición instrumental er se le transcribirá como medquic_er.

Ahora, la traducción se presentará en tres renglones. En el primero, irá el texto en mochica, segmentado de acuerdo con los criterios arriba esbozados. En un segundo renglón, irá la traducción en castellano palabra por palabra. Como la base de la traducción es el lexema, una barra diagonal / dividirá los lexemas y mediante guiones se detallarán vía abreviaturas la información gramatical que está en el mochica. Por ejemplo, a med-quic le corresponde la traducción 'oreja', pero a su vez cuenta con un morfema de no posesión (ausente en español) que abreviamos como $\neg$ PS. De esta manera, entre barras, pondremos en el segundo renglón: /oreja-ᄀPS/. En el tercer renglón, transcribiremos, ya sin ninguna indicación gramatical, una traducción libre al castellano, la cual procuraremos que sea lo más literal posible.

Al momento de proponer las oraciones traducidas con arreglo a lo expresado líneas arriba, comentaremos lo más relevante de las frases en cuestión, a efectos de darle fundamento a nuestra propuesta y hacer lo más explícitas posibles las razones que justifican nuestra traducción.

\section{Abreviaturas}

Las abreviaturas que contienen información gramatical del texto a analizar son las siguientes: $1^{\mathrm{a}}=$ primera persona, $3^{\mathrm{a}}=$ tercera persona, $\mathrm{ADV}=$ adverbializador, $\mathrm{CO}=$ concordancia de objeto, $\mathrm{COP}=$ verbo copulativo, $\mathrm{DET}$ = determinante, $\mathrm{EU}$ = eufónico, FIN = finalidad, $\mathrm{G}$ = genitivo, INS = instrumental, LOC = locativo, $\mathrm{N}=$ nominalizador, $\mathrm{PAS}=$ pasiva, $\mathrm{PL}=$ plural, $\mathrm{PR}=$ pronombre, $\mathrm{PS}=$ posesión, $\neg \mathrm{PS}=$ no posesión, $\mathrm{PT}=$ pretérito, $\mathrm{S}=$ singular, $\mathrm{VT}=$ vocal temática. Los paréntesis angulados $<>$ hacen referencia a las formas ortográficas. 


\section{Análisis}

El texto comienza señalando la existencia de cinco sentidos en el cuerpo humano y luego enumera cada uno de ellos, para dar paso a un pequeño diálogo al respecto, en el que se inquiere por la naturaleza de los sentidos y con qué finalidad nos los ha dado dios. Así, la primera oración es la siguiente:

\section{Exllmætzh-o_f ærquic-ær chi-çæ-r} cinco-EU/3a-S-COP/cuerpo-ᄀPS-G/ser-N-PS

Cinco son los sentidos del cuerpo.

En esta frase, se encuentra una de las formas del verbo copulativo. Éste se representa a través de la consonante $f$, la cual es la forma elidida de fe. En la segunda línea, hemos designado al verbo copulativo por medio de la abreviatura COP. La oración está en singular, pese a referirse a entidades en plural, como los sentidos. La concordancia no era obligatoria en mochica. El numeral exllmætzh 'cinco' tiene una vocal final o que aparece sintagmáticamente para marcar el final de un constituyente. Hemos rotulado esta vocal con la abreviatura EU, correspondiente a eufónico, aun cuando estimamos que dicho rótulo es de carácter provisional. Con todo, compárese ese numeral con la siguiente frase posposicional <Exllmætzh xang nico>. La o final ya no aparece al término del numeral exllmætzh 'cinco', sino al final de la posposición nic que es el núcleo de dicho constituyente. La distribución exacta de la o determinada sintácticamente supone la traducción de todos los textos mochicas, a efectos de realizar un estudio riguroso.

La frase nominal <ærquicær chiçær> la hemos traducido como 'sentidos del cuerpo'. El nombre <ærquicær> está en el genitivo de no posesión. Se analiza como ærquic + ær 'del cuerpo'. Hemos traducido el nombre <chiçær> como 'sentido', pues globalmente se puede inferir tal significado. Sin embargo, el nombre <chiçær> puede analizarse como chi 'ser' + çæ 'morfema nominalizador' + $r$ 'morfema de posesión'. Así, una traducción literal de <ærquicær chiçær> sería 'el ser del cuerpo'. Existen otros contextos, en los que este nombre derivado de verbo adquiere otros 
sentidos que no se derivan del análisis de su forma. Es preciso conocer mejor todos los contextos en los que se usa esta palabra para caracterizarla de mejor manera.

En lo que sigue hay cinco oraciones copulativas, enumeradas por frases donde aparece un numeral. Cada oración corresponde a cada uno de los sentidos. Comentaremos lo más destacado que observemos en las frases en cuestión.

Lech-o çio fe: ñoc-çæ-c.

Primero-EU/ 3a-S-PR/3a-S-COP/vista-N-7PS

Esto primero es: la vista

La frase se inicia con el primer numeral ordinal, el cual proviene de la palabra lecu 'cabeza'. Obviamente se trata de una metáfora. Este numeral se encuentra regido por el pronombre de tercera persona singular çio. La presencia de la vocal o nos muestra que el constituyente formado por leç está gobernado por çio. Efectivamente, uno de los contextos de aparición de esta vocal es al final de los complementos (adjetivos, nombres, deícticos, frases posposicionales) de las frases nominales, ya sea nombres o pronombres, marcando tanto límite del constituyente, como la rección. La oración copulativa es una suerte de ecuación que tiene, de un lado, el pronombre çio y, de otro, un nombre ñocçæc 'vista', derivado de un verbo de percepción ñoc 'ver', en su forma no poseída.

Pac xa-ng_nic-o çio fe: næm-a-çæ-c dos/puesto-G/LOC-EU/3a-S-PR/3a-S-COP/oído-VT-N_PS

Esto en segundo lugar es: el oído.

En esta segunda frase copulativa, se nota un cambio en la forma del numeral ordinal. En la anterior oración, simplemente se echaba mano del numeral lec $\mu$, pero para expresar los ordinales superiores a la unidad se recurre a una frase posposicional gobernada por el locativo nic, el cual rige un nombre $\langle x a\rangle$ 'turno' (o en otros contextos 'vez') que porta la marca de genitivo $\langle n g\rangle$. El nombre $x a$ expresa orden en una sucesión y funciona como una suerte de cuantificador. De ahí que se use la forma abreviada de 
los numerales correspondientes al 'dos', al 'tres' y al 'cuatro', a saber: pac, çoc y noc; en vez de la formas completas de estos numerales: aput, çopæt y nopæt ${ }^{1}$. Ahora bien, toda la frase posposicional $<$ Pac xang nico $>$ está regida por el pronombre çio como lo muestra la $o$ al final de dicho constituyente ${ }^{2}$. Esto se repetirá en los demás numerales, así que ya no llamaremos la atención sobre este hecho. En cuanto al nombre <næmaçæc> 'oído', al igual que en el caso anterior, éste deriva de un verbo cuya raíz es næm 'oír'. Lo curioso de este nombre es que para su formación requiere de una vocal temática $a$, de manera tal que se analiza del siguiente modo: næm 'oír' $+a$ 'vocal temática' + çæ 'morfema nominalizador' + c 'morfema de no posesión'. La presencia de esta vocal temática nos hace postular que la forma correcta del morfema nominalizador sería el infijo: -(V)çx-, donde la $\mathrm{V}$ representa una vocal temática opcional. La exacta determinación de la distribución de la vocal temática está sujeta al análisis de todas las formas nominalizadas que existan en el mochica, lo cual nuevamente supone la traducción de todos los textos de esta lengua.

Çoc xa-ng_nic-o çio fe: oiz-çæ-c.

tres/puesto-G/LOC-EU/3a-S-PR/3ª-S-COP/olfato-N-aPS

Esto en tercer lugar es: el olfato.

Esta oración tiene la misma estructura que la anterior. Tan sólo difiere en que el nombre oizçæc 'olfato', derivado del verbo *oiz 'oler', carece de vocal temática. Algo curioso con respecto a este verbo es que Middendorf (1892: 89) proporciona para el verbo 'oler' la raíz ōj, acompañada de la primera persona: ōjeiñ, la forma no finita de propósito: ōjnäm, y una forma no finita que dicho autor llama de supino: ōjäd.

Noc xa-ng_nic-o çio fe: ssap_æc-o cutzho chi-çæ-c.

cuatro/puesto-G/LOC-EU/3a-S-PR/3a-S-COP/boca־PS /LOC$\mathrm{EU} /$ gusto-N $\neg \mathrm{PS}$

Esto en cuarto lugar es: el gusto en la boca.

1 Sobre estas formas abreviadas de los numerales, véase Salas 2008a.

2 Similar análisis se encuentra en Salas (2008b: 335).

Bol. Acad. peru. leng. 48(48), 2009 
Cuando se menciona al sentido correspondiente al gusto se utiliza una frase compleja <ssapæco cutzho chiçæc>, la cual requiere ser analizada. La palabra ssapæco es la contracción de ssap 'boca', la posposición de locativo nic y la vocal o que indica que dicho constituyente está regido por $\langle c u t z h o>$. Con todo, la traducción de la frase es 'en la boca'. La frase <cutzho chiçæc > sería el equivalente de 'gusto' por su sentido general. Después de todo, cada uno de las oraciones enumeradas corresponde a un sentido. La palabra cutzho podría tener relación con aquella que proporciona De la Carrera (1644: 153) como cutzhio con el significado de 'chicha'. Si tal relación hubiese existido, una traducción literal de 〈ssapæco cutzho chiçæc〉 sería 'ser chicha en la boca', lo que designaría al sentido del gusto.

Exllmætzh xa-ng nic-o çio fe: cof-çæ-c. cinco/ puesto-G/LOC-EU/3a-S-PR/3a-S-COP/tacto-N-2PS Esto en quinto lugar es: el tacto.

Esta oración al igual que las cuatro anteriores comparte la misma estructura. El interés que ella puede tener radica en el nombre cofçæc 'tacto'. Al igual que en los otros casos, estamos ante un nombre derivado de verbo. Específicamente, se trataría de un hipotético verbo ${ }^{*}$ cof. Tal verbo debe haber sido de tipo psicológico. Sus posibles significados deben de acercarse a conceptos como 'sentir' o 'palpar'.

P. Ech æng, ame mo exllmætzh-o ærquic-ær chi-çæ-r? qué/3a-S-COP/asi/DET/cinco-EU/cuerpo-aPS-G/ser-N-PS ¿Qué son así estos cinco sentidos del cuerpo?

En esta pregunta hay dos elementos gramaticales que vale la pena destacar. En primer lugar, la vocal del verbo copulativo, que típicamente es ang se transforma cuando está en una construcción interrogativa, volviéndose $æ(æ n g)$. En segundo lugar, hay una extensa frase nominal $<$ mo exllmætzho ærquicær chiçær> que permite constar el orden dentro del sintagma nominal. Torero (2002: 347) afirmaba que el orden de los determinantes que precedían al núcleo nominal lo hacían en el siguiente orden: demostrativo, cuantificador, numeral, adjetivo y posesor. En efecto, el demostrativo mo antecede al numeral exllmætzho y éste a la frase en genitivo ærquicær, que a su vez antecede al núcleo. Ahora, la frase 
nominal ærquicær 'del cuerpo' no encuentra un lugar en el orden que menciona Torero. Del mismo modo, hay que decir que el mochica carecía de 'posesores', pues para expresar la posesión se utilizaba pronombres en genitivo. El orden formulado por Torero debería, en primer lugar, reemplazar el espacio para 'posesor' por uno para 'genitivo'; y, en segundo lugar, someterse a revisión teniendo en cuenta un corpus mayor, extraído de los textos que se vienen traduciendo.

R. Mo ærquic-ær chi-çæ-r fe: locu_er-o aqu-i-çæ-c:

DET/cuerpo-PS-G/ser-N-PS/3a-S-COP/ojo-PS/INS-EU/ mirada-VT-N ᄀPS

Estos sentidos del cuerpo son: la mirada con el ojo

La respuesta a esta interrogante se divide en cinco partes en las que se detalla el órgano del cuerpo con el que se produce la percepción. Para esto se usa la posposición instrumental er, a la cual se le adjunta una vocal $o$ que marca la frontera de dicho constituyente. Como se ve la posposición er no requiere de la morfología de genitivo y se adjunta directamente a la forma poseída loc $\mu$. Otro detalle que hay que resaltar es que para nombrar al sentido de la visión se usa el nombre aquiçæc, en vez de ñoç̧æc. Lo más probable es que los verbos de los que derivan estos nombres hayan sido sinónimos.

aieçæn fe, med-quic_er-o næm-a-çæ-c:

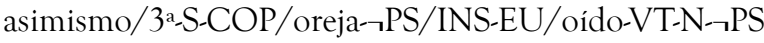

Asimismo es el oído con la oreja.

Aquí la partícula instrumental se aplica a la forma no poseída medquic, en vez de la forma poseída medeng (Carrera 1644: 177), lo cual demuestra que la combinatoria de las posposiciones podía seleccionar nombres en cualquiera de sus dos formas, a saber: poseídos y no poseídos.

aieçæn fe, fon_er-o oiz-çæ-c:

asimismo/3a-S-COP/nariz-PS/INS-EU/olfato-N-7PS

Asimismo es el olfato con la nariz. 
Algo similar a lo que ocurre con loc $\mu$, es lo que pasa con $\langle$ fonero $\rangle$. De la Carrera (1644: 5-6) menciona que la forma no poseída de este nombre es frenquic, mientras que enuncia (1644: 177) la forma poseída como: fon, foneio. El morfema instrumental se sufija directamente a la forma poseída, sin mediar la morfología de genitivo.

aieçæn fe, ssap_æc-o cutzho chi-çæ-c:

asimismo/3a-S-COP/boca-7PS/LOC-EU/gusto-N־PS

Asimismo es el gusto en la boca.

En este caso, ya en la enumeración de los sentidos se había estipulado el órgano a través del cual se podía percibir la sensación del gusto.

aieçæn fe mæcua_ro cof-çæ-c.

asimismo/3a-S-COP/mano-7PS/INS-EU/tacto-N־PS

Asimismo es el tacto con la mano.

La palabra <mæcuaro> es la fusión de la forma no poseída de mæcнa 'mano' (Carrera 1644: 178) y la posposición instrumental er, cuya vocal se fusionó con la vocal de la raíz, produciendo una construcción de orden fijo que era aprendida de memoria y, por tanto, listada en el lexicón como una excepción. Lo que acontece es que el encuentro de vocales en mochica se resolvía a través de diversos mecanismos como la inserción de consonantes o la fusión vocálica. De la Carrera (1644: 122) es explícito a propósito de esta situación con respecto a la partícula instrumental er: "Y aduiertese, que acauando el ablativo en vocal, se le añade estas dos letras ng, para con la preposicion er, como çiong er, lactung er, \&c. y dizese mæcuar, con elegancia." Así, haciendo una reconstrucción de la forma <mæcuaro>, tendremos que se obtiene a partir de la raíz mæcнa 'mano' y el instrumental (e)r, cuya vocal ponemos entre paréntesis para mostrar que fue suprimida al momento de la fusión. A este morfema instrumental se le ha añadido una vocal $o$ que indica que esta frase se encontraba regida por el nombre <cofçæc> 'tacto'.

P. Ech_pæn-o_ix piy-c-æm-æd-o Dios-i mo exllmætzh-o ærquic-ær chi-çæ-r? qué/para-EU/1a-PL/dar-CO-PAS-PT-EU/dios-G/DET/cinco- 


\section{EU/cuerpo-PS-G/ser-N-PS}

¿Para qué nos han sido dados por dios estos cinco sentidos del cuerpo?

La oración se inicia con una palabra de pregunta ech 'qué' regida por una posposición -pæn 'para' a la que se le adjunta la vocal o que marca el final de dicho constituyente. Ahora, a esta frase posposicional se le adjunta como clítico en superficie la flexión $\langle(e) i x\rangle$ que fusiona su primera vocal con la $o$ del otro constituyente, resultando solamente $\langle i x\rangle$. Hemos podido constatar a lo largo de la lectura del Arte de la lengua yunga que cuando se dan encuentros vocálicos entre la o de límite de constituyente y otras vocales se cumple que la o permanece y las otras vocales se suprimen.

En esta pregunta, salta a la vista, a su vez, la construcción en pasiva de un verbo con dos objetos. La oración en activa sería: 'Dios nos ha dado estos cinco sentidos del cuerpo'. La forma conjugada 〈ix piycæmædo> puede segmentarse de la siguiente manera: $i x$ ' 1 a plural' + piy 'dar' $+c$ 'concordancia de objeto' $+æ m$ 'pasiva' $+æ d$ 'pretérito' $+o$ 'partícula eufónica' que limita el constituyente. En esta oración se da una situación que no se produce en castellano, por eso, la traducción que hemos hecho resulta un poco forzada. En castellano, el objeto animado de los verbos con dos objetos siempre es indirecto y no puede ocupar la posición de sujeto de pasiva. La pasiva de verbos con dos objetos del mochica es como la pasiva con dos objetos del inglés, lengua en la que el objeto animado sí puede ocupar la posición de sujeto en la pasiva. Ante una oración activa como: Somebody gave the police the information, es posible realizar una pasiva de la siguiente forma: The police were given the information. Esto se produce porque los objetos animados pueden ser objetos directos en inglés. Nótese que la oración anterior tampoco recibe una traducción literal en castellano, porque los objetos animados en verbos ditransitivos del español siempre son indirectos y requieren de la preposición $a .{ }^{*}$ Los policías fueron dados la información es una oración agramatical. Una traducción correcta mantendría topicalizado el objeto indirecto, pero haría la concordancia con el objeto directo, tal como lo reclama la gramática castellana: A los policias les fue dada la información. Cuando en inglés el objeto animado es indirecto no se puede poner como sujeto pasiva, sino que es el objeto 
inanimado el que ocupa en la pasiva la posición de sujeto, al ser el objeto directo del verbo: The information was given to the police. Esta oración, a diferencia de la anterior, sí puede ser traducida literalmente al castellano, donde los objetos inanimados de los verbos ditransitivos siempre son el objeto directo: La información fue dada a los policias.

Esta construcción en pasiva con verbos de dos objetos del mochica se repite en la gramática. Así, en el Arte de la lengua yunga (Carrera 1644: 6) se encuentra la oración xllaxllad iñ llopcæm, que el autor traduce como: Mi plata me hurtaron, aun cuando el verbo mochica esté en primera persona. Esto nos muestra que la glosa castellana está adaptada a la lengua. La traducción literal sería: * Soy hurtado mi plata que tendría como activa la estructura: Alguien me hurto mi plata. La solución de Fernando de la Carrera nos parece acertada. Al sujeto de la pasiva en mochica se le pronominaliza como objeto indirecto en la traducción al castellano y al verbo se le hace concordar con el objeto directo animado. En este caso particular, está en plural, porque se trata de un agente indefinido.

Continuando con el mochica, la concordancia de objeto $c$ hace referencia a <mo exllmætzho ærquicær chiçær> 'estos cinco sentidos del cuerpo'. El morfema de pasiva æm extrae la primera persona plural de la posición de objeto, pese a ser animado, a la posición de sujeto. El sujeto de la activa se vuelve agente de la pasiva en caso genitivo: Diosi. El morfema æed es de tiempo pretérito y no ofrece mayores dificultades. Una traducción literal de la oración mochica, materia de análisis, sería: * Para qué hemos sido dados por dios estos cinco sentidos del cuerpo?, la cual es agramatical en castellano. Una mejor traducción haría concordar el verbo con el objeto directo, es decir, 'estos cinco sentidos' y pronominalizaría al sujeto como objeto indirecto: ¿Para qué nos han sido dados por dios estos cinco sentidos del cuerpo?

R. Izçæc-na çiong_er Dios mæcha læc-næm, çio çæn seruir læc-næm. todo-ADV/3aPR/INS/dios/adoración/hacer-FIN/3aPR/CONJ/ servir/hacer-FIN 
Para adorar con todos ellos a dios, y para servirlo.

En esta respuesta, hay dos detalles que merecen ser comentados. El primero es la estructura de las cláusulas de finalidad y el segundo, la referencia de los pronombres. Las dos cláusulas finales están gobernadas por la forma verbal no finita: læcnæm que proviene del verbo loc $\sim$ loqu. De la Carrera (1644: 147) dice: "Este verbo tiene el significado conforme lo que significa el accusatiuo, ò adverbio que se llega" y da un ejemplo como "confessar eiñ loc" que permite ver cómo era que los verbos del castellano se podian integrar en la estructura verbal del mochica. De igual manera, ejemplos (1644: 149) como læpæcoiñ confesar 'suelo confesarme' o læpæcoz caf 'sueles trabajar' permiten ver el cambio de la vocal de loc, cuando se le adjuntaban ciertos sufijos. Un ejemplo de la forma læcnæm con el sufijo de finalidad (Carrera 1644: 221) se encuentra en el sexto mandamiento, mostrando un caso más de armonía vocálica. Sobre este punto, véase Cerrón-Palomino (1995: 145-148) en el capítulo VII, sección 7.14.

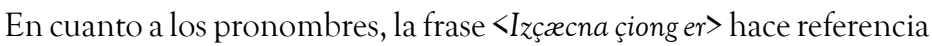
a los cinco sentidos. La pluralidad de la referencia se ve confirmada por el cuantificador izçæc. De la Carrera (1644: 14) mencionaba que "la razon porque los Indios no vsan el numero plural, es porque al singular, le allegan vn nombre adiectiuo de muchedumbre". Nótese que entre la partícula er y el pronombre çio está $\langle n g\rangle$. De la Carrera (1644: 20) advierte que "quando es instrumental el ablativo, haze. çiong. er." Atribuimos esto a la tendencia del mochica a evitar encuentros vocálicos. En este punto, disentimos de nuestro colega Hovdhaugen (2004: 55), quien considera que la posposición er marcaba con caso oblicuo a çio. Pensamos que la inserción de $\langle n g\rangle$ no tiene relación con la morfología de caso. El hecho de que uno de los alomorfos del genitivo tenga la forma ng no implica la atribución automática de dicha forma a dicho caso. Consideramos que la explicación de este fenómeno va por el lado de la estructura de la sílaba mochica, antes que por la asignación de caso. Por lo que hace al pronombre çio de la segunda cláusula, su referencia es dios. Esto lo comprobamos haciéndonos la pregunta de para servir a quién se nos han dado los cinco sentido. Así, la referencia del primer y segundo çio es disjunta. 


\section{Conclusión}

El análisis de textos permite al investigador encontrarse con fenómenos que de otra manera no se manifestarían si es que uno se ciñe al léxico de la lengua. En algunos casos, la comprensión definitiva de ciertas estructuras demanda la traducción global del corpus con el que se cuenta, el cual, huelga decirlo, está lejos de ser auscultado a cabalidad. A guisa de conclusión, ofrecemos las traducciones al castellano del texto mochica sobre los cinco sentidos corporales:

Cinco son los sentidos del cuerpo. Esto primero es: la vista. Esto en segundo lugar es: el oído. Esto en tercer lugar es: el olfato. Esto en cuarto lugar es: el gusto en la boca. Esto en quinto lugar es: el tacto.

¿Qué son así estos cinco sentidos del cuerpo?

Estos sentidos del cuerpo son: la mirada con el ojo, asimismo es el oído con la oreja, asimismo es el olfato con la nariz, asimismo es el gusto en la boca, asimismo es el tacto con la mano.

¿Para qué nos han sido dados por dios estos cinco sentidos del cuerpo?

Para adorar con todos ellos a dios, y para servirlo.

\section{BIBLIOGRAFÍA}

CARRERA DAZA, Fernando de la [1604- después de 1665]. ARTE DE LA LENGVA YVNGA DE LOS VALLES del Obispado de Truxillo del Peru, con un Confessonario, y todas las Oraciones Christianas, traducidas en la lengua, y otras cosas. Lima, Ioseph de Contreras, 1644.

CERRÓN-PALOMINO, Rodolfo Marcial [1940]. La lengua de Naimlap (reconstrucción y obsolescencia del mochica). Lima, Fondo Editorial de la PUCP. 1995. 
HOVDHAUGEN, Even [1941]. Mochica (= Languages of the World/Materials 433). Munich, LINCOM GmbH, 2004.

MIDDENDORF, Ernst Wilhelm [1830-1908]. Das Muchik oder die ChimuSprache (= Die einheimischen Sprachen Perus, sechster Band). Leipzig, F. A. Brockhaus, 1892.

SALAS GARCÍA, José Antonio [1975]. "Formas ligadas en los numerales del mochica”. Lexis, volumen XXXII, número 1, pp. 147158. 2008a.

"La Señal de la Cruz en la lengua mochica". Lexis, volumen XXXII, número 2, pp. 321-345, 2008b.

TERCER CONCILIO LIMENSE. DOCTRINA CHRISTIANA, Y CATECISMO PARA INSTRVCCION de los Indios, y de las de mas personas, que han de ser enseñadas en nuestra sancta Fé. CON VN CONFESSIONARIO, Y OTRAS COSAS necessarias para los que doctrinan. Edición facsimilar de la de Antonio Ricardo. Lima, Ediciones Copé de Petroperú, [1584] 1984.

TORERO FERNÁNDEZ DE CÓRDOVA, Alfredo Augusto [19302004]. Idiomas de los Andes. Lingüística e historia (= Travaux de l'Institut Français d'Études Andines 162). Lima: IFEA \& Editorial Horizonte, 2002.

\section{Correspondencia:}

\section{José Antonio Salas García}

Lingüista de profesión, autor del Diccionario mochica-castellano, castellano mochica. joseantoniosalas-blogspot.com 